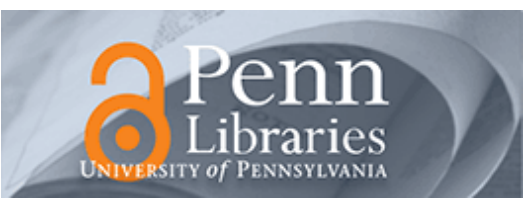

University of Pennsylvania

ScholarlyCommons

3-1-1995

\title{
MediSim: Simulated Medical Corpsmen and Casualties for Medical Forces Planning and Training
}

\author{
Norman I. Badler \\ University of Pennsylvania, badler@seas.upenn.edu \\ John R. Clarke \\ Medical College of Pennsylvania \\ Michael J. Hollick \\ University of Pennsylvania \\ Evangelos Kokkevis \\ University of Pennsylvania \\ Dimitris Metaxas

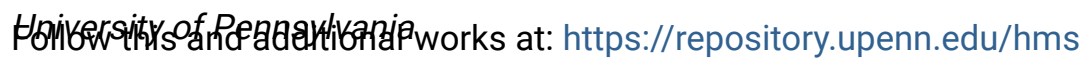

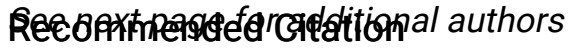 \\ Badler, N. I., Clarke, J. R., Hollick, M. J., Kokkevis, E., Metaxas, D., Bindiganavale, R., Webber, B. L., Chi, D. M., Foster, N., Ogunyemi, O., \& Kaye, J. (1995). MediSim: Simulated Medical Corpsmen and Casualties for Medical Forces Planning and Training. Retrieved from https://repository.upenn.edu/hms/37}

Copyright 1995 IEEE. Reprinted from Proceedings of the National Forum on Military Telemedicine On-Line Today, 1995, March 1995, pages 21-28.

Publisher URL: http://dx.doi.org/10.1109/MTOL.1995.504523

This material is posted here with permission of the IEEE. Such permission of the IEEE does not in any way imply IEEE endorsement of any of the University of Pennsylvania's products or services. Internal or personal use of this material is permitted. However, permission to reprint/republish this material for advertising or promotional purposes or for creating new collective works for resale or redistribution must be obtained from the IEEE by writing to pubs-permissions@ieee.org. By choosing to view this document, you agree to all provisions of the copyright laws protecting it.

This paper is posted at ScholarlyCommons. https://repository.upenn.edu/hms/37

For more information, please contact repository@pobox.upenn.edu. 


\title{
MediSim: Simulated Medical Corpsmen and Casualties for Medical Forces Planning and Training
}

\author{
Abstract \\ The MediSim system extends virtual environments (both local and network) to represent simulated \\ medical personnel interacting with simulated casualties. Our technology fosters dual-use applications \\ involving planning, training, and evaluation of both medical corpsmen and civilian EMTs. Behaviors and \\ behavioral control are being developed for the medical corpsmen that will enable their actions on the \\ digital battlefield to conform to both military practice and medical protocols. From situationally- \\ appropriate injury models, a set of physical and behavioral manifestations in a simulated casualty will be \\ determined and portrayed on a three-dimensional body.
}

\section{Keywords}

injury models, medical procedures, computer simulation, virtual environments, physics-based modeling, medical decision support

\section{Comments}

Copyright 1995 IEEE. Reprinted from Proceedings of the National Forum on Military Telemedicine On-Line Today, 1995, March 1995, pages 21-28.

Publisher URL: http://dx.doi.org/10.1109/MTOL.1995.504523

This material is posted here with permission of the IEEE. Such permission of the IEEE does not in any way imply IEEE endorsement of any of the University of Pennsylvania's products or services. Internal or personal use of this material is permitted. However, permission to reprint/republish this material for advertising or promotional purposes or for creating new collective works for resale or redistribution must be obtained from the IEEE by writing to pubs-permissions@ieee.org. By choosing to view this document, you agree to all provisions of the copyright laws protecting it.

\section{Author(s)}

Norman I. Badler, John R. Clarke, Michael J. Hollick, Evangelos Kokkevis, Dimitris Metaxas, Ramamani Bindiganavale, Bonnie L. Webber, Diane M. Chi, Nick Foster, Omolola Ogunyemi, and Jonathan Kaye 


\title{
MediSim: \\ SimulatedMedical Corpsmen and Casualties for Medical Forces Planning and Training*
}

\author{
Norman I. Badler \\ John R. Clarke, M.D. \\ Michael J. Hollick \\ Evangelos Kokkevis \\ Dimitri N. Metaxas \\ Rama Bindiganavale
}

\author{
Bonnie L. Webber \\ Diane M. Chi \\ Nick Foster \\ Omolola Ogunyemi \\ Jonathan Kaye
}

\author{
Center for Human Modeling and Simulation \\ Department of Computer and Information Science \\ University of Pennsylvania \\ Philadelphia, PA 19104-6389
}

\begin{abstract}
The MediSim system extends virtual environments (both local and network) to represent simulated medical personnel interacting with simulated casualties. Our technology fosters dual-use applications involving planning, training, and evaluation of both medical corpsmen and civilian EMTs. Behaviors and behavioral control are being developed for the medical corpsmen that will enable their actions on the digital battlefield to conform to both military practice and medical protocols. From situationally-appropriate injury models, a set of physical and behavioral manifestations in a simulated casualty will be determined and portrayed on a three-dimensional body.

Keywords: Injury models, medical procedures, computer simulation, virtual environments, physicsbased modeling, medical decision support.

\section{Introduction}

The MediSim system extends virtual environments to represent simulated medical personnel interacting with simulated casualties. Fig. 1 diagrams the major components of the MediSim system.

\footnotetext{
${ }^{0}$ Appeared in The National Forum: Military Telemedicine On-Line Today. Research, Practice and Opportunities, IEEE
} Computer Society Press, 1995.
\end{abstract}

- Existing human models are being extended to provide close-range simulated casualties. Casualties must show medically significant features such as face, skeletal structures, and internal organs. Wound and injury types are being coupled probabilistically to the simulated battlefield. Casualties must appear sufficiently real in terms of both physical behavior and appearance, in order to motivate the trainee and his avatar to expeditiously but carefully provide medical intervention. The Jack ${ }^{\circledR}[3]$ human model is being extended with capabilities for appropriate injury display. A stealth human instructor can create simulated injuries and patient responses to treatment while monitoring the training exercise.

- The semi-automated medical corpsman (SAM) must be able to demonstrate rescue and medical assessment procedures as a model for the trainee or to function as an independent agent during a simulation. The SAM must demonstrate sufficient trauma care skills and practices in conformance with corpsman or EMT protocols to render reasonable and responsible simulated medical care. The Jack human model behavioral set is being augmented with a repertoire of relevant corpsman behaviors such as taking vital signs to confirm and/or augment PSM data, immobilizing suspected fractures, and stabilizing and trans- 


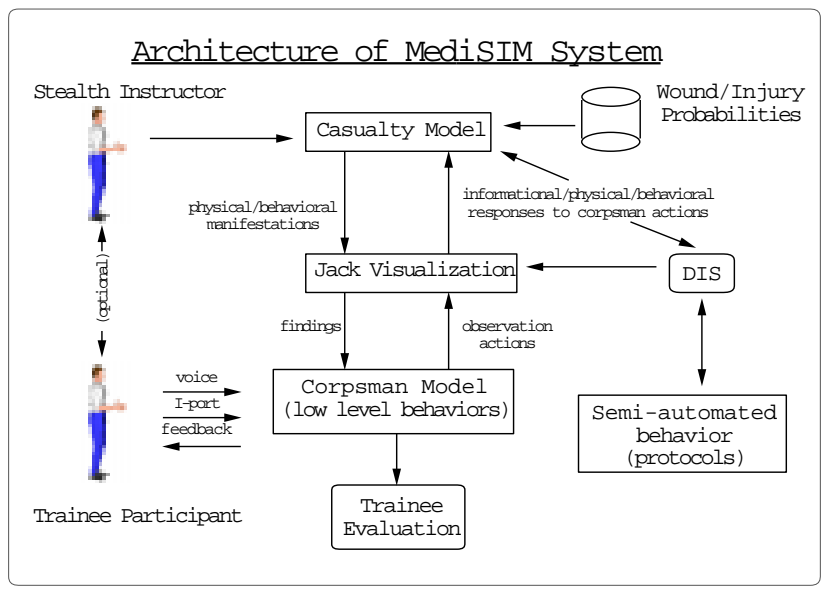

Figure 1: Architecture of Proposed MediSim System

porting casualties with signs and symptoms of shock. Task ordering will be determined by established medical corps or EMT protocols [5, 13].

- Virtual Environment technology will insure the feeling of presence in the battlefield environment [14, 16] (Fig. 2). A VE embedding (avatar) of a corpsman must be produced, with physical control over navigation and voice control over medical procedures, allowing the corpsman to direct his/her avatar as if instructing a knowledgeable assistant.

- Evaluation measures will assess both quantitative (timing, amount of movement, change in patient's physiological state) and qualitative (care quality, effectiveness) aspects of interactive corpsman training.

MediSim offers a number of significant advances:

- Synthetic generation and visualization of wounds and injuries, along with their concomitant physical, physiological, and behavioral manifestations.

- Model-based visualizations of injuries and vital signs that trigger corpsman decision-making and behaviors implemented as a hierarchy of behavior nets.

- Task-based coordination of either multiple synthetic human figures or combinations of synthetic figures and VR avatars.

- Communication channel between the trainee and the avatar modeled in an effective VE embedding.

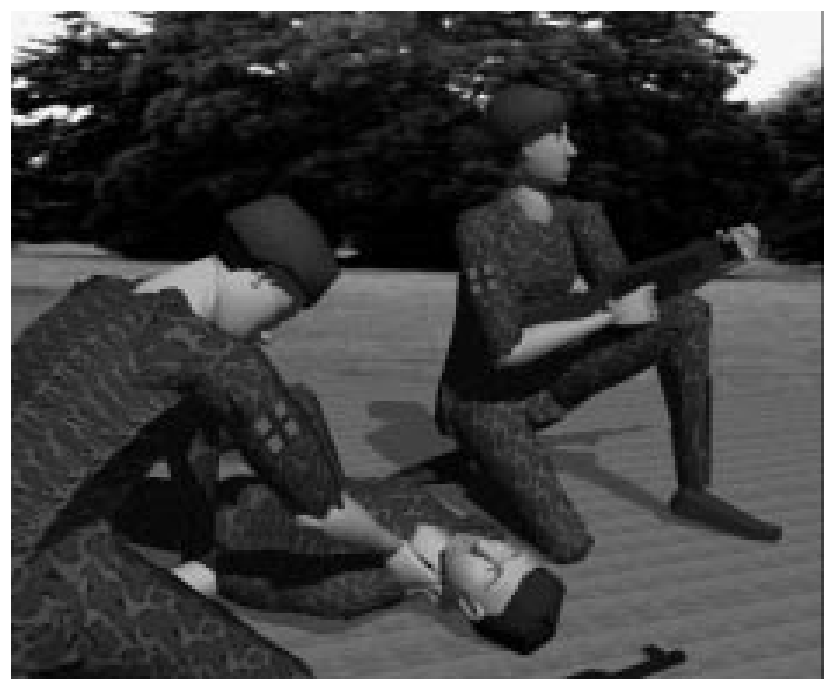

Figure 2: Scene in the Battlefield Environment

- The use of a "stealth" instructor to create, monitor, and effect injuries and dictate the outcomes of procedures or treatment response, places the MediSim project within the highly successful paradigm of flight simulation but entails major innovation in the stealth's user interface.

- A dual-use but "military-ready" system is to be designed from the start with DIS compatibility where possible. Both civilian EMT and military medical corpsmen procedures will be modeled within the same extensible framework.

In this paper, we first present an overview of the MediSim System and then discuss a prototype realtime implementation. Next, we discuss our model of the wounded soldier, including the generation of penetrating injuries, casualty simulation, local wound simulation, and physics-based modeling of medical procedures. We conclude with a discussion of concurrent work in medical decision support and developments in integrated anatomical and physiological modeling.

\section{The MediSim System}

Our MediSim medical corpsman system consists of several simulated individuals: one or more casualties with battlefield or situation appropriate wounds, and human agents representing simulated medical corpsmen who carry out procedures on the casualties. An agent may either be a semi-autonomous medic $(S A M)$ or else an intentionally correlated embodiment (avatar) of a real participant's actions and commands. 
In the latter case, the participant experiences the casualty care situation with its concomitant dangers and challenges and may thus participate through the avatar as a second virtual agent observing, assisting, and commanding the SAM.

Casualties under corpsman care should display appropriate signs and manifestations of battlefield injury, for purposes of corpsman training. Physiological state information is available by visual inspection, appropriate query, or correct performance of an assessment procedure. The medical corpsman behavioral repertoire needs to include protocol and procedures for rendering on-site emergency assessment and care for a selection of such injuries. Consequently, the physical and physiological effects and consequences of those procedures on the simulated casualty and his injuries are portrayed. During the course of the training activity, the casualty's wounds and injuries as well as his response to medical procedures can be probabilistically simulated - or controlled by a "behind the scenes" or "stealth" instructor analogous to the experienced human trainer creating emergency situations for pilots during flight simulation.

The simulated medical corpsman will be able to act semi-autonomously as a SAM, or through a live participant in an I-Port Virtual Environment interface as an avatar. The SAM will be able to navigate the digital nanoterrain of the battlefield, requesting and/or responding to vital sign information transmitted by personnel status monitors (PSM). Protocols will direct the SAM to select the next casualty to attend to, using PSM, GPS, access route and threat data. Proximity to the casualty will enable physiological data to become available, triggering appropriate assessment, initial management, and evacuation procedures. The physical interactions between SAM and casualty will be portrayed through real-time behaviors computed automatically by the underlying human figure simulation system.

For the live corpsman participant, the avatar will conform to input behaviors in carrying out navigation, and on verbal command will execute various behaviors to carry out assessment, management, and evacuation. The participant is both observer and trainee, making decisions and giving commands to guide the physically capable, obedient, but passive avatar. Quantitative evaluation measures will be examined to gauge the efficacy of virtual training environments for medical personnel.

\subsection{Prototype Real-Time Implementa- tion}

Early versions of portions of MediSim have been demonstrated. In these configurations, pre-rendered medical animation sequences were added to the JackML motion library. NPSNet (from the Naval Postgraduate School [14]) used the JackML to generate human motions involving typical combat-oriented activities, as well as specialized medical treatment animations. The participant in the exercises interacted with the environment by using the Sarcos I-Port, a VR mobility simulator.

This system was tied into a standard Distributed Interactive Simulation (DIS) network. In this way, medic treatment scenarios could be performed in an environment populated by external DIS entities (although the medical animations themselves were not rendered on standard DIS image generators). Flags needed for medical animation were stored in unused segments of standard DIS Protocol Data Units; as this system evolves, new methods of transporting this data will be developed.

\subsection{Modeling the Wounded Soldier}

Our basic approach to casualty modeling is to (1) generate a set of wounds and determine appropriate injuries on a simulated casualty either based on Army probabilities (conditioned on weaponry, battlefield layout, etc.) or through penetration path models (Section 2.2.1) ; (2) map them to likely findings through information in our trauma management decision support system, TraumAid (see Section 3.1); and (3) instantiate their physical and behavioral manifestations in a human figure simulated in Jack and their physiological manifestations in simulated PSM data.

Given the weaponry in use, battlefield layout, etc., conditional probabilities specify the likelihood of different wound(s). Given particular wounds, further conditional probabilities specify the types of injuries a casualty is likely to suffer. We will use these probabilities to generate simulated casualties. Alternatively, the stealth instructor can generate specific casualty types and responses (cf. Section 2.2.3).

Rules used in TraumAid to reason from findings and test results forward to diagnostic and therapeutic goals can be inverted to map penetrating injuries of the abdomen and chest, back to findings that in connection with wound specifications give evidence for those injuries. The problem that test results will not be available can be solved in the same way as in 
TraumAID's retrospective evaluation [8]: for diagnostic tests that were not performed during actual care, a realistic "default" database associated the likely result of each test with each particular discharge diagnosis.

Findings can manifest through a casualty's physical appearance or his behavior. Physical appearance can be observed visually (e.g. distended abdomen, evisceration) or aurally (e.g., decreased breath sounds, stridor). Observation may be passive or require activity on the part of the corpsman (e.g., tactile probing for signs of tenderness and/or guarding). Behaviors that give evidence of injuries or degree of injury include confusion, level of response to commands, gasping muscle spasms, etc.

Although the current TraumAid system only covers penetrating injuries to the chest and abdomen, small Traum Aid rule sets for upper and lower extremity injuries developed for the Navy's Submarine Center at Groton [7] will allow us to generate at least a subset of appropriate findings for such injuries as well.

By the end of the current year, we expect to demonstrate casualties exhibiting physical manifestations of selected integument and musculo-skeletal injuries. During the second year, casualties will exhibit both physical and behavioral manifestations of additional integument, musculo-skeletal and internal injuries; for example, swelling, pale skin color, exposed viscera, breathing anomalies, muscle spasms, or facial expressions.

\subsubsection{Modeling Penetrating Injuries}

Determining the anatomical and physiological impact of penetrating wounds involves relating knowledge of anatomy with possible projectile penetration paths. Our penetration path module assists in evaluating penetrating injuries to the chest and abdomen, and complements MediSim's injury model. Delp [10] addresses penetrating injuries to the legs.

Penetrating injuries caused by gunshot or stab wounds are modeled using a rotatable $3 \mathrm{D}$ graphical model of the torso with the appropriate anatomical structures. External wounds corresponding to either gunshot or stab wounds can be entered onto any location on the surface of the $3 \mathrm{D}$ body model. In the case of ballistic injuries, the presence of multiple entry and exit wounds complicates the process of determining which organs have been injured.

A combinatorial analysis of the surface wounds leads to various penetration path hypotheses. For any given pair of wounds, we define a wound path space as the space of possible paths a bullet may have taken from one wound to the other. Similarly, for a sin- gle external wound, a wound path space describes the space of possible trajectories from the wound to a bullet lodged in the body. A penetration path hypothesis may consist of one or more wound path spaces, depending on the number of wounds.

Once all the possible pairings of wounds for a given set of penetrating injuries have been determined, the system establishes which anatomical structures are affected for each wound path space. By displaying each pairing of penetration path and injured organs for a given set of wounds, the system provides a visual cue to their consequences. This can help in bridging the gap between knowledge of the anatomy involved in a particular injury and the physiological manifestations associated with that injury.

\subsubsection{Casualty Simulation}

Casualty simulation begins with the specification of injuries sustained by the victim. Currently, the modeled injuries are (1) chest injury resulting in a tension pneumothorax and (2) abdominal injury resulting in abdominal bleeding, hemothorax, and pericardial tamponade. The simulation displays plots of the victim's vital signs over time. The plotted values of the vital signs use the rankings given in the Trauma Scale [6] endorsed by the American Trauma Society for estimating the injury severity. The trauma scale reflects basic assessments of the respiratory, circulatory, and central nervous systems. Fig. 3 shows the Trauma Scale rating system.

A Jack human figure portrays the physical and behavioral manifestations of its injuries and responds appropriately to commands representing various diagnostic and therapeutic procedures. The following diagnostic queries result in a text response written to the screen: respiratory-rate?, blood-pressure?, capillary-refill?, name?, id?. Visual responses from the Jack figure result from assessing respiratory expansion, eye opening ability, and motor response. These commands include: open-your-eyes, apply-sternal-pressure, move-your-foot, and squeeze-my-hand. The user may also use the following commands for administering treatments: needle-aspiration, give-fluids, and occlusive-dressing. The commands for assessing the situation are: are-you-ok?, what-happened?, do-you-have-pain?, and where-does-it-hurt?. The commands and queries are currently not implemented as procedures carried out by the (semi-)autonomous Jack medic, but rather text input commands to the interface which invoke an appropriate patient response. The simulation will 


\begin{tabular}{lll} 
Respiratory & $10-24 / \mathrm{min}$ & 4 \\
& $24-35 / \mathrm{min}$ & 3 \\
& $36 / \mathrm{min}$ or greater & 2 \\
& $1-9 / \mathrm{min}$ & 1 \\
& None & 0 \\
\hline Respiratory & Normal & 1 \\
Expansion & Retractive & 0 \\
\hline Systolic & $90 \mathrm{mmHg}$ or greater & 4 \\
Blood & $70-89 \mathrm{mmHg}$ & 3 \\
Pressure & $50-69 \mathrm{mmHg}$ & 2 \\
& $0-49 \mathrm{mmHg}$ & 1 \\
& No Pulse & 0 \\
\hline Capillary & Normal & 2 \\
Refill & Delayed & 1 \\
& None & 0 \\
\hline \multirow{3}{*}{ Glasgow Coma Scale } & \\
Eye & Spontaneous & 4 \\
Opening & To Voice & 3 \\
& To Pain & 2 \\
& None & 1 \\
\hline Verbal & Oriented & 5 \\
Response & Confused & 4 \\
& Inappropriate Words & 3 \\
& Incomprehensive Words & 2 \\
& None & 1 \\
\hline Motor & Obeys Commands & 6 \\
Response & Localized Pain & 5 \\
& Withdraw Pain & 4 \\
& Flexion (pain) & 3 \\
& Extension (pain) & 2 \\
\hline & None & 1 \\
\hline & &
\end{tabular}

Figure 3: Trauma Scale

be extended to display additional physical and behavioral manifestations; for example, distended neck veins, cyanosis, thrashing, and other general expressions of pain.

The model is built using Parallel Transition Networks (PaT-Nets), a package for creating and running parallel state-machines [2, 4]. There are two main types of networks that compose the casualty model:

- Vital sign networks which generate the visual effects of changes in trauma scale measurements and respond to user input, and

- Injury networks that specify the physiological changes resulting from specific medical conditions.
Each modeled vital sign has a network containing methods to produce appropriate visual and textual responses to user commands reflecting the patient's current state (trauma score values). A controller network ensures that the values remain in their valid ranges.

Physiological conditions that result from injury are modeled as individual PaT-Nets. These networks specify the transitions in the vital sign controller network for the trauma scale measurements. The patient's condition, reflected in the trauma score, is set to deteriorate or improve at the appropriate times, depending on the performed therapeutic intervention (or lack thereof). Currently, the modeled conditions include tension pneumothorax, hemothorax, pericardial tamponade, and abdominal bleeding. A casualty may receive multiple conditions as result of injury. For instance, a penetrating abdominal wound may result in hemothorax, pericardial tamponade, and abdominal bleeding. When multiple conditions occur, the lowest value of each trauma scale value is used as the overall value. The current networks were built based on estimates from one of the authors, an experienced trauma surgeon (Clarke).

\subsubsection{Case Study: Tension Pneumothorax}

Fig. 4 shows the tension pneumothorax and needle aspiration PaT-Nets. The machine starts in the Base Node. A clock starts at the time of injury. After specific time intervals $(1,3,5,8,12$, and 15 minutes), the PaT-Net transitions to a new state where the appropriate vital signs are decremented. Thereafter, the PaT-Net immediately returns to the Base Node. If the patient is given a needle aspiration, the tension pneumothorax PaT-Net exits and spawns a needle aspiration PaT-Net. The needle aspiration PaT-Net starts in its Base Node. At one and two minute intervals, it transitions to a new state and increments the appropriate vital sign values. Fig. 5 shows the plot of the vital signs reflecting the Central Nervous System when the patient develops a tension pneumothorax and receives no treatment. Fig. 6 shows the Central Nervous System plot when the patient receives a needle aspiration 9 minutes after the time of injury.

\subsection{Localized wound simulation}

Wound simulation is considered important in the training of first response emergency teams for two reasons;

1. The physical appearance and behavior over time of the wound site are vital clues for diagnosis. 


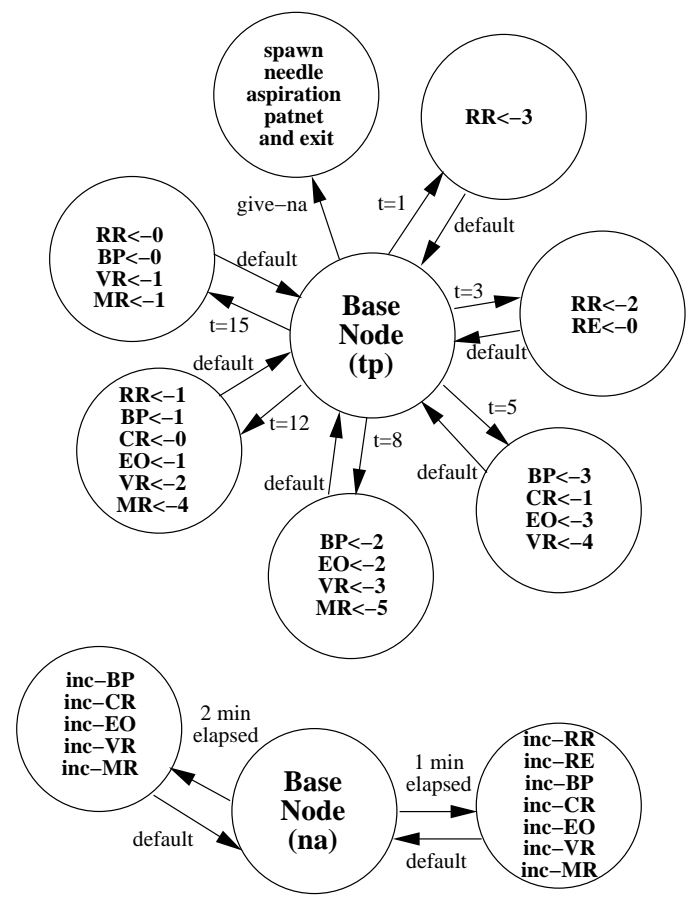

Figure 4: Tension Pneumothorax and Needle Aspiration PaT-Nets

2. The size, shape, and location of wounds influence the rates at which physiological changes occur in the casualty.

The goal of this part of the project is to combine an accurate model of the wounding mechanism, i.e. the damage caused by a projectile, with a visual portrayal of the victim's localized physical response. Bleeding is one of the most important aspects of that response because blood loss not only impacts the extent of the injury but may also cause secondary trauma such as paralysis. A system for modeling and animating liquid phenomena [12] is being developed to simulate internal and external bleeding due to penetrative wounds. The method is based around the Navier-Stokes equations which can be used to describe the motion of a viscous fluid. This model has been extended to give realistic behavior to blood including clotting and water bridging. Where possible, computationally expensive parts of the fluid calculation have been simplified to give the high frame rate necessary for the real-time requirement of the MediSim application.

\subsection{Physics-based Modeling of Medical Procedures}

Physical realism plays an important role in the simulation of the medical corpsmen procedures. The

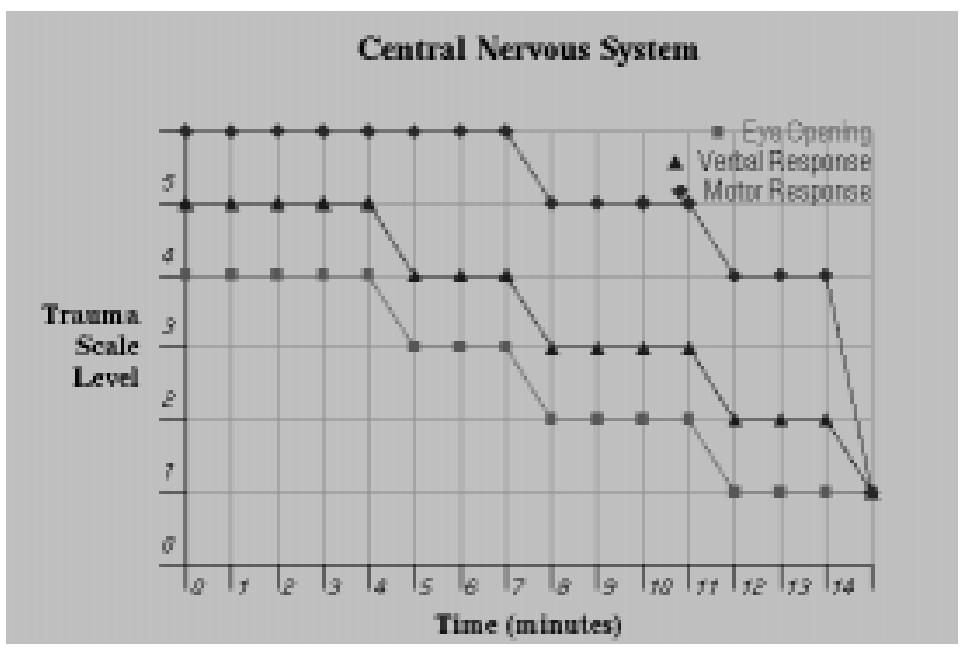

Figure 5: Tension Pneumothorax Plot - No Treatment

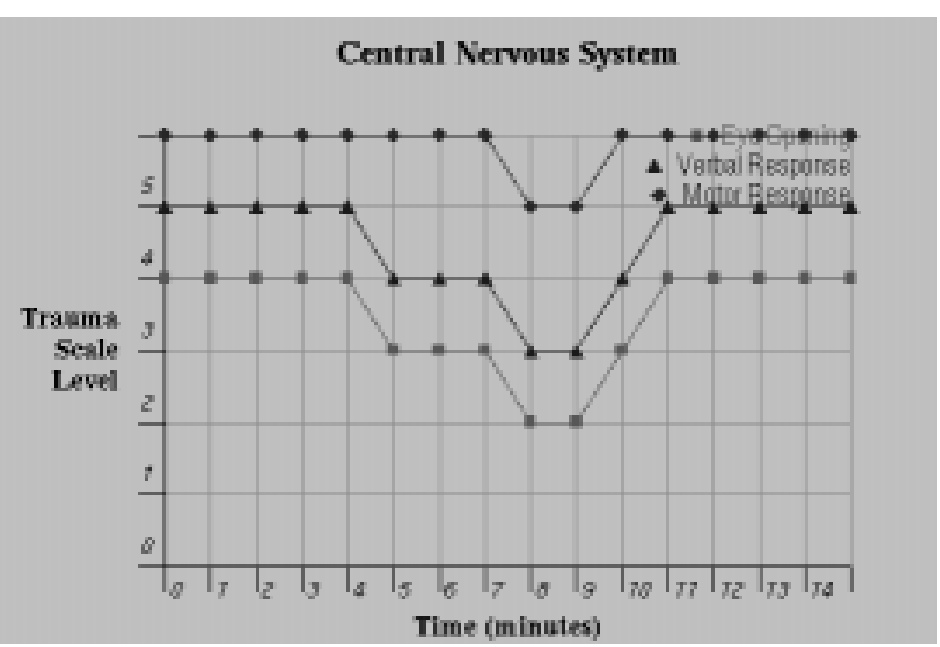

Figure 6: Tension Pneumothorax Plot - Needle Aspiration at 9 minutes 
quality of the motion animation can be enhanced by adding dynamical properties to the humans and objects present in the scenes. After assigning realistic mass and inertia to both the body parts of the injured humans and the medical instruments and tools, natural looking motion can be generated though a dynamic simulator. The corpsman can interact with the environment and perform the specified operations by applying (mainly through his hands) external forces directly to the injured person. The effect of these forces will automatically be computed by the dynamic simulator and the resulting motion of the parts and objects involved will be both physically correct and convincing. The advantages offered from dynamic simulation are not limited only to the visual realism of the animation. Given accurate strength limits for the human body muscles and joints, the feasibility of certain operations that the corpsman performs can be judged. If more force to achieve a certain result (such as moving the injured body) is needed than the corpsman can apply then a request for extra help can be issued. A fast recursive dynamic simulator [11] specially suited for the efficient animation of articulated figures is applied to compute the motions in real time. The inertial properties of objects involved in the scenes can either be automatically inferred by assuming a preset mass density for each object or can be set off-line by a user. Dynamic simulation adds to the realism of simulated procedures, provides quantitative measures for the feasibility of operations on the injured humans, and automates the process of creating new simulation scenarios.

Patient response will conform dynamically and probabilistically to a set of implemented injury types. Using Metaxas' techniques of physics-based modeling and dynamic deformations [15], we will be able to do some dynamic computation of the motion of unsupported body parts and restraint of distended soft organs/tissues.

The casualty model generates simulations of various battlefield injuries. The model produces appropriate physical, physiological, and behavioral responses to both injuries and medical intervention.

\section{Related Activities}

\subsection{Medical Decision Support and Treat- ment Planning}

We have developed and retrospectively validated a decision-support system for the initial definitive management of multiple trauma (penetrating injuries of the chest and abdomen) in Emergency Centers [8, 18]. Deployment in the Emergency Center at the Medical College of Pennsylvania for the purpose of prospective evaluation began in April 1995. An earlier version of the TraumAid system was adapted to use by medical corpsmen on submarines and extended to cover basic care of injuries to the extremities as well [7].

TraumAID was designed to complement the coverage of ATLS procedures [1] and conform to the information needs of local, state and national trauma registries. We are now following developments in the recently established Trauma Care Information Management Systems (TCIMS) TRP consortium, whose goal is to apply telecommunications, information and decision support technologies to improving the speed and effectiveness of pre-hospital trauma care. We see that through interfacing with TCIMS, information collected pre-hospitally can be used to reduce TraumAID's in-hospital data collection needs and position it to provide decision support for definitive management as soon as the patient enters the Emergency Center.

\subsection{Integrated Anatomical and Physio- logical Modeling}

The TrauMAP project aims to link physiological models that express mechanical behavior (and ultimately chemical and neurological functions) with a visually- and structurally-accurate anatomical model. Work to date has focussed on modeling respiratory mechanics, in order to demonstrate both quiet, normal breathing and pathologies in the form of pneumothoraces. If one were only interested in such demonstrations per se, scripted animation might suffice. However, since the aim is to simulate and ultimately predict interactions among body systems, physical object models are needed that respond to and transmit forces. Because the anatomical components are principally soft tissue, that response must include object deformation. Thus, the work to date has involved integrating a 3D deformable simulation of the lungs and thoracic space with physiological process equations, expressed as ordinary differential equations. This work is described in more detail in [9].

\section{Acknowledgments}

This work is being undertaken in conjunction with Sharon Stansfield of Sandia National Laboratories and Michael Zyda and David Pratt of the Naval Postgraduate School. This research is partially supported by ARPA Biomed Program DAMD17-94-J4486; NLM N01-LM-4-3515; DMSO DAAH04-94- 
G-0402; U.S. Air Force DEPTH through Hughes Missile Systems F33615-91-C-0001; ARO DURIP DAAH04-95-1-0023; Army AASERT DAAH04-94-G0220; ARPA AASERT DAAH04-94-G-0362; and NSF CISE CDA88-22719.

\section{References}

[1] American College of Surgeons, Advanced Trauma Life Support, Chicago: American College of Surgeons, 1984.

[2] Badler, N. and Becket, W., "Integrated Behavioral Agent Architecture," Proc. 3rd Conf. on Computer Generated Forces and Behavior Representation, Orlando, FL, pp. 57-68, March 1993.

[3] Badler, N., Phillips, C. and Webber, B., Simulating Humans: Computer Graphics, Animation, and Control, Oxford: Oxford University Press, 1993.

[4] Badler, N., Webber, B., Becket, W., Geib, C., Moore, M., Pelachaud, C., Reich, B., and Stone, M., "Planning for Animation," in D. Thalmann and N. Magnanat-Thalmann (eds.), Computer Animation, New York: Prentice Hall Inc., 1995.

[5] Brady, Emergency Care, 5th edition.

[6] Champion, H.R., Sacco, W.J., Carnazzo, A.J., et. al., "Trauma Score," Critical Care Med. 9(9), pp. 672-676, 1981 .

[7] Clarke, J.R., Niv, M., Webber, B.L., Fisherkeller, K., Southerland, D.G. and Ryack, B., "TraumAid: A Decision Aid for Managing Trauma at Various Levels of Resources," Proc. 13th Annual Symp. on Computer Applications in Medical Care (SCAMC), Washington D.C., pp. 1005-1006 (abstract), November 1989.

[8] Clarke, J.R., Rymon, R., Webber, B., Hayward, C., Santora, T., Wagner, D. and Ruffin, A., "The Importance of Planning in the Provision of Medical Care," Medical Decision Making 13(4), p. 383 (abstract), October-December 1993.

[9] DeCarlo, D., Kaye, J., Metaxas, D., Clarke, J.R., Webber, B. and Badler, N., "Integrating Anatomy and Physiology for Behavior Modeling," in Medicine Meets Virtual Reality III: Interactive Technology and the New Paradigm for Healthcare, R.M. Satava, K. Morgan, H.B.
Sieburg, et al eds., Amsterdam: IOS Press, pp. 81-87, 1995 .

[10] Delp, S., "Battlefield Casualty Care Using the Virtual Human," this volume.

[11] Featherstone, R., "The Calculation of Robot Dynamics Using Articulated-Body Inertias," International Journal of Robotics Research, 2(1), Spring 1983.

[12] Foster, N. and Metaxas, D., "Realistic Animation of Liquids," Submitted to GMIP, 1995.

[13] Henry, M. and Stapleton, E., EMT Prehospital Care, Philadelphia: W.B. Saunders Co., 1992.

[14] Macedonia, M.R., Zyda, M.J., Pratt, D.R., Barham, P.T. and Zeswitz, P., "NPSNET: A Network Software Architecture for Large-Scale Virtual Environments," Presence: Teleoperators and Virtual Environments 3(4), pp. 265-287, 1994.

[15] Metaxas, D. and Koh, E., "Efficient Shape Representation Using Deformable Models with Locally Adaptive Finite Elements," Geometric Methods in Computer Vision II, B.C. Vemuri (ed.), Proc. SPIE 2031, pp. 160-171, San Diego, CA, July 1993.

[16] Stansfield, S.A., "Distributed Virtual Reality Simulation System for Situational Training," Presence: Teleoperators and Virtual Environments 3(4), pp. 360-366, 1994.

[17] U. S. Army Medical Department (AMEDD) Videotapes, Perform Patient Assessment, Fort Sam Houston, TX, November 1993.

[18] Webber, B., Rymon, R. and Clarke, J.R., "Flexible Support for Trauma Management through Goal-directed Reasoning and Planning," Artificial Intelligence in Medicine 4(2), pp. 145-163, April 1992. 